\title{
A multicenter survey of enteroscopy for the diagnosis of intestinal follicular lymphoma
}

\author{
MASAYA IWAMURO $^{1}$, HIROYUKI OKADA ${ }^{2}$, SEIJI KAWANO ${ }^{2}$, JUNJI SHIODE ${ }^{3}$, RYUTA TAKENAKA ${ }^{4}$, \\ ATSUSHI IMAGAWA ${ }^{5}$, TOMOKI INABA ${ }^{6}$, SEIYU SUZUKI ${ }^{7}$, MAMORU NISHIMURA $^{8}$, MOTOWO MIZUNO ${ }^{9}$, \\ MASASHI ARAKI ${ }^{10}$, TOMOHIKO MANNAMI $^{11}$, TORU UEKI ${ }^{12}$, HARUHIKO KOBASHI $^{13}$, \\ HARUKA FUKATSU ${ }^{14}$, SHOUICHI TANAKA ${ }^{15}$, AKIYOSHI OMOTO ${ }^{16}$, YOSHINARI KAWAI ${ }^{17}$, \\ TAKASHI KITAGAWA ${ }^{18}$, TATSUYA TOYOKAWA ${ }^{19}$, KATSUYOSHI TAKATA ${ }^{20}$, \\ TADASHI YOSHINO $^{20}$, AKINOBU TAKAKI ${ }^{21}$ and KAZUHIDE YAMAMOTO ${ }^{21}$
}

\footnotetext{
${ }^{1}$ Department of Molecular Hepatology, Okayama University Graduate School of Medicine, Dentistry and Pharmaceutical Sciences; ${ }^{2}$ Department of Endoscopy, Okayama University Hospital, Okayama 700-8558; ${ }^{3}$ Department of Internal Medicine, Okayama Saiseikai General Hospital, Okayama 700-8511; ${ }^{4}$ Department of Internal Medicine, Tsuyama Chuo Hospital,

Tsuyama 708-0841; ${ }^{5}$ Department of Gastroenterology, Mitoyo General Hospital, Kanonji 769-1695;

${ }^{6}$ Department of Gastroenterology, Kagawa Prefectural Central Hospital, Takamatsu 760-8557;

${ }^{7}$ Department of Internal Medicine, Sumitomo Besshi Hospital, Niihama 792-8543; ${ }^{8}$ Department of Internal Medicine, Okayama City Hospital, Okayama 700-8557; ${ }^{9}$ Department of Internal Medicine, Hiroshima City Hospital,

Hiroshima 730-8518; ${ }^{10}$ Department of Internal Medicine, Kagawa Rosai Hospital, Marugame 763-8502;

${ }^{11}$ Department of Internal Medicine, Chugoku Central Hospital, Fukuyama 720-0001; ${ }^{12}$ Department of Internal Medicine,

Fukuyama City Hospital, Fukuyama 721-8511; ${ }^{13}$ Department of Hepatology, Japanese Red Cross Okayama Hospital,

Okayama 700-8607; ${ }^{14}$ Department of Internal Medicine, St. Mary's Hospital, Himeji 670-0801;

${ }^{15}$ Department of Gastroenterology, Iwakuni Clinical Center, Iwakuni 740-8510; ${ }^{16}$ Department of Internal Medicine, Ako Central Hospital, Ako 678-0241; ${ }^{17}$ Department of Gastroenterology, Onomichi Municipal Hospital, Onomichi 722-8503;

${ }^{18}$ Department of Surgery, Kitagawa Hospital, Okayama 709-0497; ${ }^{19}$ Department of Gastroenterology,

Fukuyama Medical Center, Fukuyama 720-8520; Departments of ${ }^{20}$ Pathology and ${ }^{21}$ Gastroenterology and Hepatology,

Okayama University Graduate School of Medicine, Dentistry and Pharmaceutical Sciences, Okayama 700-8558, Japan
}

Received October 7, 2014; Accepted April 27, 2015

DOI: $10.3892 / 01.2015 .3251$

\begin{abstract}
The importance of enteroscopy examinations to investigate the entire length of the small intestines has been emphasized in follicular lymphoma patients with intestinal involvement. The aim of the present study was to determine the current state of enteroscopy examinations, including the performance rate, and the prevalence of small intestinal lesions in a patient population in Japan. A retrospective multicenter survey of 17 institutions collected the case information
\end{abstract}

Correspondence to: Dr Masaya Iwamuro, Department of Molecular Hepatology, Okayama University Graduate School of Medicine, Dentistry and Pharmaceutical Sciences, 2-5-1 Shikata-cho, Kita-Ku, Okayama 700-8558, Japan

E-mail: iwamuromasaya@yahoo.co.jp

Key words: intestinal follicular lymphoma, duodenal neoplasms, double-balloon enteroscopy, video capsule enteroscopy, small intestine of 110 follicular lymphoma patients with gastrointestinal involvement. The results of the enteroscopy examinations were reviewed, and in order to identify potential factors affecting the performance rate of enteroscopy, patient gender, age at lymphoma diagnosis, histopathological grade, clinical stage, the date of the initial diagnosis and the annual volume of enteroscopy at the institution were compared between the patients who underwent one or more enteroscopy procedures and the patients who did not undergo enteroscopy. A total of 34 patients $(30.9 \%)$ underwent enteroscopy, and 24 of these (70.6\%) presented with involvement in the jejunum and/or ileum. It was found that more patients diagnosed in recent years and more patients treated at an ultra-high volume institution ( $\geq 101$ enteroscopy examinations/year) underwent an enteroscopy. In conclusion, although the prevalence of small intestinal lesions was high $(70.6 \%)$ in the follicular lymphoma patients presenting with intestinal involvement, the performance rate of enteroscopy was only $30.9 \%$, and thus the majority of the patients have not undergone enteroscopy examinations. Further investigation is required to define the clinical significance of enteroscopy at the initial diagnostic work-up and during the follow-up period of these patients. 


\section{Introduction}

Follicular lymphoma is the most common subtype of indolent non-Hodgkin's lymphoma. Widespread nodal involvement is a typical presentation of this disease, with the majority of patients presenting with Ann Arbor stage III or IV, whereas only $5-10 \%$ of patients present with early stage I or II (1-3). Follicular lymphoma arising in the intestine has recently been drawing attention for its distinct clinical features, which are different from their nodal counterpart. Several studies have suggested that the majority of patients with intestinal follicular lymphoma present with localized disease and show favorable clinical courses (3-5).

Intestinal follicular lymphomas were initially introduced as a disease predominantly involving the duodenum (6). The representative macroscopic features of the duodenal lesions are multiple small, whitish polypoid lesions (5). The detection of such duodenal lesions by esophagogastroduodenoscopy is the initial diagnostic clue in the cases of the majority of the patients with this disease entity. Since the development of novel enteroscopy devices such as double-balloon enteroscopy and video capsule enteroscopy, recent studies have revealed the frequent involvement of the jejunum and ileum in these patients. Among follicular lymphoma patients presenting with intestinal involvement, the reported percentage of cases with multiple follicular lymphoma lesions in the jejunum and/or ileum has ranged from 66.7 to $100 \%$ (3,7-11).

These results emphasize the importance of enteroscopy examinations to precisely evaluate the extent of the intestinal lesions in follicular lymphoma patients. However, the majority of the previous studies were conducted in a single tertiary-care center, and to the best of our knowledge, the number of patients with this disease entity who underwent enteroscopy examinations in actual clinical settings has never been determined. A retrospective multicenter survey was therefore conducted to reveal the present status of enteroscopy examinations among follicular lymphoma patients presenting with intestinal involvement. The purposes of the present study were to determine the current state of enteroscopy examinations, including the performance rate, and to determine the prevalence of small intestinal lesions in a patient population in Japan.

\section{Patients and methods}

Patients. A database search performed at the Department of Pathology at the Okayama University Graduate School of Medicine, Dentistry and Pharmaceutical Sciences (Okayama, Japan) identified 110 follicular lymphoma patients with gastrointestinal involvement who were treated at Okayama University Hospital or one of 16 collaborating institutions (listed in the Acknowledgements section) between July 1990 and October 2013. A subset of the 110 patients examined also participated in a number of our previous studies $(4,6,12-19)$.

The diagnosis of follicular lymphomas was made according to World Health Organization (WHO) classifications $(1,20)$. A histological diagnosis was based on morphological and immunophenotypical analyses of endoscopically biopsied specimens or surgically resected specimens. Histopathological grading was also determined according to WHO criteria (1). Patients with grade 3 follicular lymphoma were excluded from this study since these cases are generally managed based on treatment strategies established for diffuse large B-cell lymphomas (1). Clinical data regarding the patients' endoscopic, radiological and biological examinations were obtained from retrospectively reviewed clinical records. The Lugano staging system for the classification of gastrointestinal lymphoma was used to determine each patient's clinical stage $(21,22)$.

The present study was approved by the Ethical Committee of the Okayama University Hospital and adhered to the Declaration of Helsinki.

Analyses. Based on the clinical records of the enrolled patients, the patients were divided into two groups: The Ent group was composed of the patients who underwent any type of enteroscopy, such as per oral/per anal double-balloon enteroscopy and video capsule enteroscopy, and the No-Ent group was comprised of the patients who had never undergone enteroscopy. First, to determine the prevalence of follicular lymphoma lesions in the small intestine and their endoscopic features, the study evaluated the Ent group patients' modalities of enteroscopy performed, the macroscopic appearance of the lesions and the affected regions of the small intestine. Subsequently, to identify potential factors affecting the performance rate of enteroscopy among follicular lymphoma patients with gastrointestinal involvement, patient gender, age at lymphoma diagnosis, histopathological grade, clinical stage, the date of the initial diagnosis and the annual volume of enteroscopy examinations at the institution where the patient was treated were evaluated.

The annual volume of enteroscopy is presented as the total number of enteroscopy examinations performed at the participating institution in 2013. The 17 institutions were then divided into the following four groups in accordance with the annual number of enteroscopies performed: Low-volume institutions, 0-25 examinations/year (9 institutions); middle-volume institutions, 26-50 examinations/year (3 institutions); high-volume institutions, 51-100 examinations/year (3 institutions); and ultra-high volume institutions, >101 examinations/year (2 institutions).

Statistical analysis. For the comparisons of the two groups, statistical analyses, including t-tests, $\chi^{2}$ tests and F-tests were performed using JMP 8.0.1 software (SAS Institute, Cary, NC, USA). $\mathrm{P}<0.05$ was considered to indicate a statistically significant difference.

\section{Results}

Among the 110 patients, 34 patients (30.9\%) had undergone one or more enteroscopy examinations at the initial diagnostic work-up and/or during the follow-up period (Table I). A total of 21 patients underwent video capsule enteroscopy, 17 patients underwent double-balloon enteroscopy, 1 patient underwent per-oral single balloon enteroscopy and 1 patient underwent per-oral push enteroscopy. A total of 6 patients underwent a video capsule enteroscopy plus a double-balloon enteroscopy. Of the 10 patients in whom the small intestine was investigated only by double-balloon enteroscopy, 3 underwent only a per-oral enteroscopy, 2 underwent only a per-anal enteroscopy and the remaining 6 underwent a per-oral plus a per-anal enteroscopy. Eventually, the entire length of the 
Table I. Prevalence and endoscopic features of follicular lymphoma lesions in the small intestine

\begin{tabular}{lc} 
Feature & No. of patients \\
\hline Total no. of patients & 34 \\
Modality of enteroscopy & 21 \\
VCE & 17 \\
DBE & 1 \\
SBE & 1 \\
Push enteroscopy & \\
Affected regions of the small intestine & 16 \\
Jejunum only & 2 \\
Ileum only & 6 \\
Jejunum plus ileum & 10 \\
None & \\
Macroscopic appearance & 22 \\
Whitish granules & \\
Ulcer & 2 \\
Stenosis & 2 \\
Ulcerative tumor & 1 \\
Wall thickening & 1 \\
\end{tabular}

a20 cases showed only whitish granular lesions. VCE, video capsule enteroscopy; DBE, double-balloon enteroscopy; SBE, single-balloon enteroscopy.

small intestine was examined in 28 patients by video capsule enteroscopy and/or the combination of per-oral plus per-anal double-balloon enteroscopy.

The enteroscopy examinations revealed only jejunal involvement in 16 patients and only ileal involvement in 2 patients. A total of 6 patients presented with small intestinal lesions in the jejunum plus the ileum, whereas 10 patients exhibited no involvement in the small intestine. Finally 24/34 patients (70.6\%) exhibited involvement in the jejunum and/or ileum. Among the 34 patients who underwent enteroscopy, 32 patients (94.1\%) presented with duodenal involvement and 22/32 patients $(68.8 \%)$ with involvement in the jejunum and/or ileum.

In terms of the macroscopic appearance, 22 patients showed whitish granules in the jejunum and/or ileum (Figs. 1 and 2). Such whitish granules, which are quite similar to the well-known features observed in duodenal follicular lymphoma $(1,5)$, were the only presenting feature in 20 patients. In the remaining 4 patients, 1 presented with an ulcerative tumor (Fig. 3), another with luminal stenosis, the third with whitish granules, wall thickening and ulcers, and the fourth with whitish granules, luminal stenosis and ulcers.

The patients were subdivided into the following two groups according to the status of their small intestinal examinations: The Ent group, i.e., the patients who underwent enteroscopy $(n=34)$, and the No-Ent group, i.e., the patients who did not undergo an enteroscopy $(n=76)$. The clinical characteristics of each group are provided in Table II. The comparisons of the two groups revealed that patient age $(\mathrm{P}=0.886)$, gender $(\mathrm{P}=1.000)$, histological grade $(\mathrm{P}=0.674)$ and clinical stage (I and II1 vs. II2 and IV; $\mathrm{P}=0.663$ ) did not differ significantly between the groups, whereas more patients diagnosed in recent years (since 2008) underwent enteroscopy examinations compared with the patients diagnosed previously (in 2007 or earlier; $\mathrm{P}<0.001$ ). Additionally, more patients treated at an ultra-high volume institution were examined by enteroscopy compared with the patients treated at other institutions (low-, middle- and high-volume institutions; $\mathrm{P}=0.003$ ).

\section{Discussion}

The primary purpose of the present study was to investigate the performance rate of enteroscopy in follicular lymphoma patients presenting with gastrointestinal involvement. To the best of our knowledge, this study is the first to reveal the performance rate in actual clinical settings. In this study, 34/110 patients (30.9\%) underwent enteroscopy examinations. The statistical analysis disclosed that the small intestines were more frequently investigated in patients diagnosed in recent years (since 2008) and in patients treated at the institutions where a considerable number of enteroscopy examinations was performed ( $\geq 101$ examinations/year). Conversely, the number of enteroscopy examinations was fewer in patients diagnosed in earlier years, particularly in 2007 or prior to this, and in patients treated at institutions where $<100$ examinations were performed per year.

In Japan, double-balloon enteroscopy was introduced in the fall of 2003 (23) and video capsule enteroscopy was introduced in the fall of 2007 (24). It is likely that the availability of enteroscopy devices at the initial staging of follicular lymphoma patients affected the performance rate of the enteroscopic examinations. Another possible reason is that the high prevalence of the intestinal lesions was recognized in 2008. However, it is noteworthy that enteroscopy examinations were not performed at the initial work-up or even in the follow-up period in 41 of the 44 patients (93.2\%) who were diagnosed in 2007 or earlier.

As aforementioned, previous studies documented that the jejunum and/or ileum was affected in 66.7 to $100 \%$ of follicular lymphoma patients presenting with intestinal involvement (3,4,7-11). The present study showed a similar prevalence, as the jejunum and/or ileum was affected in $24 / 34$ patients $(70.6 \%)$. Consequently, all the follicular lymphoma patients presenting with intestinal involvement can be regarded as candidates for enteroscopy examinations, regardless of the availability of enteroscopy devices in the institution and regardless of the phase of the patient's clinical course (i.e., at the initial work-up or during follow-up period).

In the present study, the jejunum and/or ileum was affected in 24 patients. Among them, 20 patients (83.3\%) developed whitish granules as the only presenting feature. Other studies have described the small intestinal lesions observed by enteroscopy as 'multiple white nodules', 'multiple small granules', 'small whitish polyps', 'whitish granular lesions' or 'polypoid lesions' $(5,8,9,11)$. The present study and previous reports indicated that the whitish granules, which are similar to the typical endoscopic features of duodenal follicular lymphoma, are the predominant morphology of the jejunal and ileal involvement as well. Nakamura et al noted that the jejunal lesions are easily identified as follicular lymphoma lesions as physiological lymphoid structures rarely exist in the jejunum, whereas ileal lesions are occasionally difficult to differentiate from physiological lymphoid follicles (11). In such cases, histological 
Table II. Clinical backgrounds of the Ent and No-Ent patients.

\begin{tabular}{|c|c|c|c|}
\hline Parameter & Enteroscopy & No enteroscopy & P-value \\
\hline No. of patients & 34 & 76 & \\
\hline Male/female, $\mathrm{n}$ & $15 / 19$ & $34 / 42$ & 1.000 \\
\hline Age at diagnosis of FL, years & $63.0 \pm 2.0$ & $63.4 \pm 1.4$ & 0.886 \\
\hline WHO grade, $\mathrm{n}$ & & & 0.674 \\
\hline Grade 1 & 31 & 72 & \\
\hline Grade 2 & 3 & 4 & \\
\hline Lugano system staging, $\mathrm{n}$ & & & 0.663 \\
\hline $\mathrm{I}$ & 19 & 39 & \\
\hline II1 & 5 & 10 & \\
\hline II2 & 3 & 4 & \\
\hline IV & 7 & 23 & \\
\hline Date of initial diagnosis, $\mathrm{n}$ & & & $<0.001^{\mathrm{a}}$ \\
\hline $1990-2003$ & 2 & 20 & \\
\hline 2004-2007 & 1 & 21 & \\
\hline 2008-present & 31 & 35 & \\
\hline Annual volume of enteroscopy at the institution, $n$ & & & $0.003^{\mathrm{b}}$ \\
\hline $0-25$ & 6 & 27 & \\
\hline $26-50$ & 3 & 6 & \\
\hline $51-100$ & 3 & 17 & \\
\hline $101+$ & 22 & 26 & \\
\hline
\end{tabular}

${ }^{\mathrm{a}} 1990-2007$ vs. 2008 -present; ${ }^{\circ} 0-100$ vs. $\geq 101$.
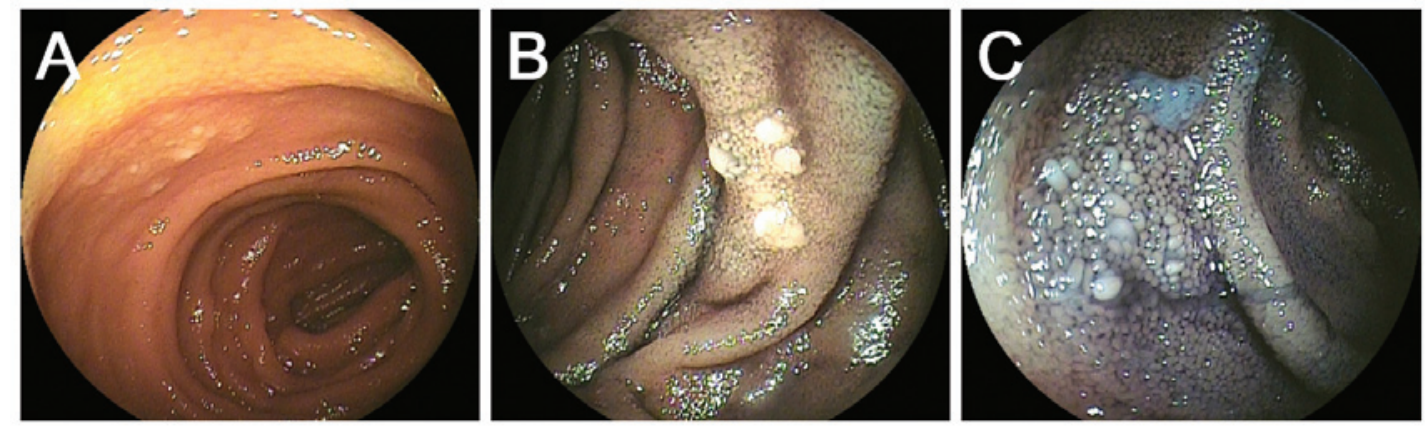

Figure 1. Images of enteroscopy results for a 73-year-old male with stage I follicular lymphoma. Per-oral double-balloon enteroscopy images showing whitish granules in the (A) duodenum and (B) jejunum. (C) The whitish granules are emphasized by indigo carmine spraying.

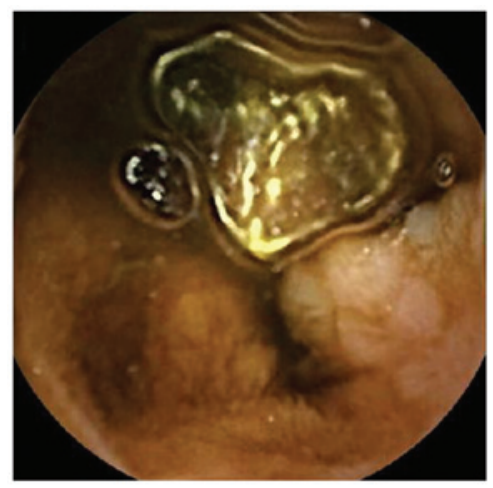

Figure 2. An image of enteroscopy results for a 61-year-old female with stage I follicular lymphoma. Video capsule enteroscopy image revealing whitish granules in the jejunum in addition to the duodenal lesions. examinations with the immunostaining of biopsied specimens are required for an accurate diagnosis.

The present study also found that 4 patients exhibited macroscopic features other than whitish granules, namely ulcerative lesions, luminal stenosis, tumorous lesions accompanied by ulcers and/or wall thickening. These morphologies were probably formed during the progression of the disease and as a result of an increased number of infiltrated lymphoma cells within the small intestinal wall $(7,25)$. Biopsy examinations are generally useful for such lesions showing atypical macroscopic features, not only for the accurate pathological diagnosis of follicular lymphomas, but also for the detection of the histological transformation from grades 1 and 2 to grade 3 , which generally facilitates the prompt initiation of treatment. 

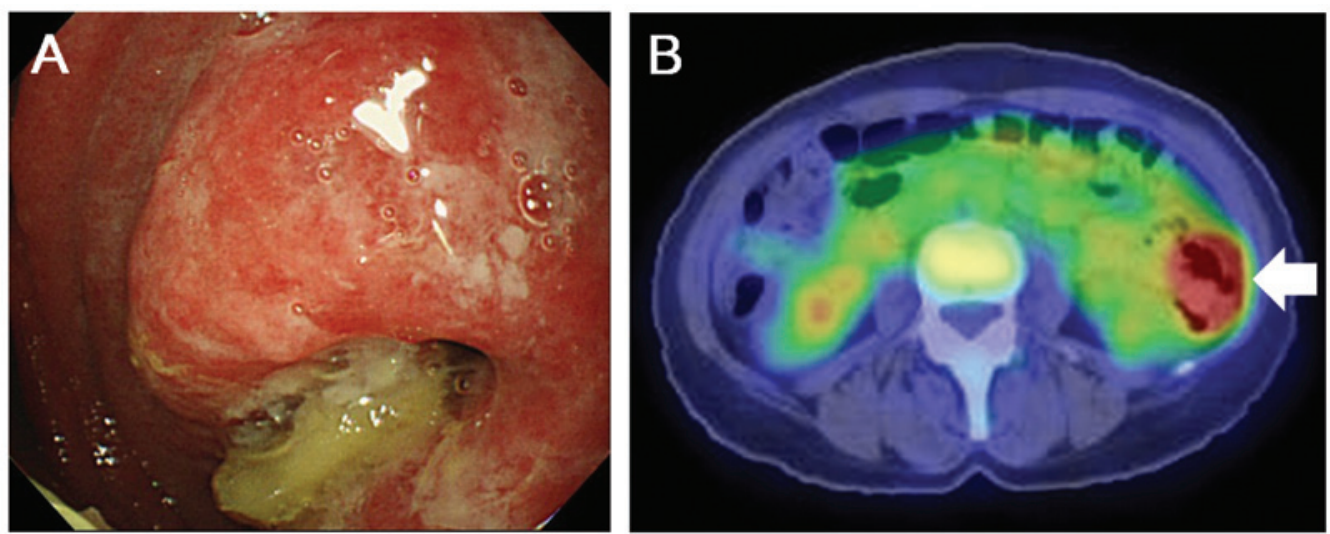

Figure 3. Images of enteroscopy results for a 49-year-old female with stage I follicular lymphoma. (A) An ulcerative tumor was observed in the jejunum by the per-oral single-balloon enteroscopy examination. This case also had duodenal involvement showing whitish granules. (B) A diffusely thickened wall was detected by computed tomography scan, and an accumulation of tracer was observed in the jejunal lesion (arrow) by positron emission tomography.

No standardized treatment strategies have been established for limited-stage gastrointestinal follicular lymphoma. The initial treatment may be radiotherapy, systemic chemotherapy, monoclonal antibody monotherapy, a combination of these therapies or a 'watch and wait' policy (3-5). Several studies have stated that the watch and wait strategy can be an acceptable initial approach due to the indolent nature of this disease entity $(2,3,12,26-28)$. On the other hand, for limited-stage follicular lymphoma of nodal origin, radiotherapy is recommended as the preferred treatment with curative potential $(2,29)$. In the study by Schmatz et al, it was reported that all the patients with primary intestinal follicular lymphoma treated by radiotherapy alone $(n=19)$ experienced complete remission, and none of these patients developed a local recurrence during a median follow-up time of 37 months (3). Radiotherapy may thus be one of the treatment options for limited-stage gastrointestinal follicular lymphoma, as well as for nodal cases. In such situations, surveillance of the jejunum and ileum prior to local treatment is particularly vital, since the majority of treatment failures occur outside the involved field of radiotherapy (30).

At the present time, double-balloon enteroscopy and video capsule enteroscopy are the two major devices that enable the direct visualization of the entire length of the small intestines. Video capsule enteroscopy is more convenient for patients than double-balloon enteroscopy; the capsule-type device is easily swallowed, and the full length of the small intestines can be examined at a single examination session. Moreover, sedation and exposure to $\mathrm{X}$-ray is not required during a video capsule enteroscopy procedure. On the other hand, although the double-balloon enteroscopy generally requires sedation, exposure to X-rays, and per-oral and per-anal examinations to observe the entire small intestine, it provides the great advantage of biopsy sampling. A biopsy is particularly valuable for the diagnostic work-up of ileal lesions of follicular lymphoma and for the detection of histological transformation, as aforementioned.

The results of the present study revealed that the majority of the patients $(76 / 110,69.1 \%)$ had not undergone enteroscopy examinations at the initial work-up and/or in the follow-up period, despite the high prevalence of small intestinal lesions among these patients. At the present time, the clinical significance of enteroscopy examinations in follicular lymphoma patients remains a subject of debate, as it has not been revealed whether or not small intestinal lesions detected by enteroscopy affect the prognoses of follicular lymphoma patients. We believe that, in follicular lymphoma patients presenting with gastrointestinal involvement (particularly in patients at limited stages), the entire gastrointestinal tract should be screened by video-capsule enteroscopy, esophagogastroduodenoscopy and colonoscopy at the initial diagnostic work-up.

Double-balloon enteroscopy is required to take biopsy samples in cases of lesions with atypical morphology or ileal lesions mimicking physiological lymphoid follicles, in order to make an accurate pathological diagnosis and to detect the histological transformation. We contend that this policy is acceptable, as the histological transformation to grade 3 follicular lymphoma generally requires the prompt initiation of treatment. Other topics to debate concerning enteroscopy examinations include whether enteroscopy should be repeated during the follow-up period and whether a biopsy is required at each enteroscopy examination to screen for transformation. Further studies should address these questions.

The present study has certain limitations. First, among the 34 patients who underwent enteroscopy, 24 patients $(70.6 \%)$ presented with jejunal and/or ileal involvement, but not all of the 34 patients had the entire small intestine examined. For example, 1 patient underwent per-oral push enteroscopy, 3 patients underwent per-oral enteroscopy only, and two patients underwent only a per-anal enteroscopy. As a result, an underestimation of the prevalence of small intestinal lesions may have occurred. Second, the enteroscopy examinations were performed under different conditions, since the patients were treated at various institutions. It is possible that differences in methodology among the participating institutions affected the detection of small intestinal lesions.

In conclusion, this multicenter survey revealed that $34 / 110(30.9 \%)$ follicular lymphoma patients presenting with intestinal involvement underwent enteroscopy examinations, and that jejunal and/or ileal involvement was frequently identified (24/34 patients, $70.6 \%)$. The clinical significance of precisely evaluating the extent of the intestinal lesions by enteroscopy should be determined hereafter. 


\section{Acknowledgements}

The authors would like to thank the following 16 collaborating institutions for providing the database information: Okayama Saiseikai General Hospital, Okayama; Tsuyama Chuo Hospital, Tsuyama; Mitoyo General Hospital, Kanonji; Kagawa Prefectural Central Hospital, Takamatsu; Sumitomo Besshi Hospital, Niihama; Okayama City Hospital, Okayama; Hiroshima City Hospital, Hiroshima; Kagawa Rosai Hospital, Marugame; Chugoku Central Hospital, Fukuyama; Fukuyama City Hospital, Fukuyama; Japanese Red Cross Okayama Hospital, Okayama; St. Mary's Hospital, Himeji; Iwakuni Clinical Center, Iwakuni; Ako Central Hospital, Ako; Onomichi Municipal Hospital, Onomichi; and Kitagawa Hospital, Okayama, Japan.

\section{References}

1. Harris NL, Swerdlow SH, Jaffe ES, et al: Follicular lymphoma. In: WHO Classification of Tumours of Haematopoietic and Lymphoid Tissues. Swerdlow SH, Campo E, Harris NL, et al (eds). IARC press, Lyon, France, pp220-226, 2008.

2. Dreyling M, Ghielmini M, Marcus R, Salles G, Vitolo U; ESMO Guidelines Working Group: Newly diagnosed and relapsed follicular lymphoma: ESMO Clinical Practice Guidelines for diagnosis, treatment and follow-up. Ann Oncol 22 (suppl 6) vi59-vi63, 2011.

3. Schmatz AI, Streubel B, Kretschmer-Chott E, et al: Primary follicular lymphoma of the duodenum is a distinct mucosal/submucosal variant of follicular lymphoma: A retrospective study of 63 cases. J Clin Oncol 29: 1445-1451, 2011.

4. Takata K, Okada H, Ohmiya N, et al: Primary gastrointestinal follicular lymphoma involving the duodenal second portion is a distinct entity: A multicenter, retrospective analysis in Japan. Cancer Sci 102: 1532-1536, 2011.

5. Yamamoto S, Nakase H, Yamashita K, et al: Gastrointestinal follicular lymphoma: Review of the literature. J Gastroenterol 45: 370-388, 2010

6. Yoshino T, Miyake K, Ichimura K, et al: Increased incidence of follicular lymphoma in the duodenum. Am J Surg Pathol 24: 688-693, 2000

7. Higuchi N, Sumida Y, Nakamura K, et al: Impact of double-balloon endoscopy on the diagnosis of jejunoileal involvement in primary intestinal follicular lymphomas: A case series. Endoscopy 41: 175-178, 2009.

8. Akamatsu T, Kaneko Y, Ota H, Miyabayashi H, Arakura N and Tanaka E: Usefulness of double balloon enteroscopy and video capsule endoscopy for the diagnosis and management of primary follicular lymphoma of the gastrointestinal tract in its early stages. Dig Endosc 22: 33-38, 2010.

9. Kodama M, Kitadai Y, Shishido T, et al: Primary follicular lymphoma of the gastrointestinal tract: A retrospective case series. Endoscopy 40: 343-346, 2008.

10. Nakamura S, Matsumoto T, Umeno J, et al: Endoscopic features of intestinal follicular lymphoma: The value of double-balloon enteroscopy. Endoscopy 39 (suppl 1): E26-E27, 2007.

11. Nakamura M, Ohmiya N, Hirooka Y, et al: Endoscopic diagnosis of follicular lymphoma with small-bowel involvement using video capsule endoscopy and double-balloon endoscopy: A case series. Endoscopy 45: 67-70, 2013.
12. TakataK, Sato Y,Nakamura N, et al: Duodenal and nodal follicular lymphomas are distinct: The former lacks activation-induced cytidine deaminase and follicular dendritic cells despite ongoing somatic hypermutations. Mod Pathol 22: 940-949, 2009.

13. Takata K, Sato Y, Nakamura N, et al: Duodenal follicular lymphoma lacks AID but expresses $\mathrm{BACH} 2$ and has memory B-cell characteristics. Mod Pathol 26: 22-31, 2013.

14. Takata K, Tanino M, Ennishi D, et al: Duodenal follicular lymphoma: Comprehensive gene expression analysis with insights into pathogenesis. Cancer Sci 105: 608-615, 2014.

15. Sato Y,Ichimura K, Tanaka T, et al: Duodenal follicular lymphomas share common characteristics with mucosa-associated lymphoid tissue lymphomas. J Clin Pathol 61: 377-381, 2008.

16. Iwamuro $\mathrm{M}$, Okada $\mathrm{H}$, Takata $\mathrm{K}$, et al: Diagnostic role of $18 \mathrm{~F}$-fluorodeoxyglucose positron emission tomography for follicular lymphoma with gastrointestinal involvement. World J Gastroenterol 18: 6427-6436, 2012.

17. Takeuchi K, Iwamuro M, Imagawa A, et al: Primary follicular lymphoma of the duodenum with erosions as atypical macroscopic features. Case Rep Med 2012: 582607, 2012.

18. Iwamuro M, Okuda M, Yumoto E, et al: Magnifying endoscopy for intestinal follicular lymphoma is helpful for prompt diagnosis. Gut Liver 7: 258-261,2013.

19. Iwamuro M, Shinagawa K, Okada H, Takata K, Yoshino T and Yamamoto K: Elevated soluble IL-2 receptor levels correlate with tumor bulk of follicular lymphomas with intestinal involvement. Clin Biochem 47: 191-195, 2014.

20. Harris NL, Jaffe ES, Diebold J, et al: World Health Organization classification of neoplastic diseases of the hematopoietic and lymphoid tissues: Report of the Clinical Advisory Committee meeting-Airlie House, Virginia, November 1997. J Clin Oncol 17: 3835-3849, 1999.

21. Zucca E, Roggero E, Bertoni F and Cavalli F: Primary extranodal non-Hodgkin's lymphomas. Part 1: Gastrointestinal, cutaneous and genitourinary lymphomas. Ann Oncol 8: 727-737, 1997.

22. Rohatiner A, d'Amore F, Coiffier B, et al: Report on a workshop convened to discuss the pathological and staging classifications of gastrointestinal tract lymphoma. Ann Oncol 5: 397-400, 1994.

23. Yamamoto H, Yano T, Kita H, Sunada K, Ido K and Sugano K: New system of double-balloon enteroscopy for diagnosis and treatment of small intestinal disorders. Gastroenterology 125: 1556-1557, 2003

24. Nakamura M, Ohmiya N, Niwa Y and Goto H: Endoscopic examination for the small bowel-video capsule endoscopy and double balloon enteroscopy. Nihon Rinsho 66: 1253-1258, 2008 (In Japanese).

25. Iddan G, Meron G, Glukhovsky A and Swain P: Wireless capsule endoscopy. Nature 405: 417, 2000.

26. Misdraji J, Harris NL, Hasserjian RP, Lauwers GY and Ferry JA: Primary follicular lymphoma of the gastrointestinal tract. Am J Surg Pathol 35: 1255-1263, 2011.

27. Campbell BA, Voss N, Woods R, et al: Long-term outcomes for patients with limited stage follicular lymphoma: Involved regional radiotherapy versus involved node radiotherapy. Cancer 116: 3797-3806, 2010.

28. Yanai S, Nakamura S, Takeshita M, et al: Translocation $\mathrm{t}(14 ; 18) / \mathrm{IGH}-\mathrm{BCL} 2$ in gastrointestinal follicular lymphoma: Correlation with clinicopathologic features in 48 patients. Cancer 117: 2467-2477, 2011.

29. Mac Manus MP and Hoppe RT: Is radiotherapy curative for stage I and II low-grade follicular lymphoma? Results of a long-term follow-up study of patients treated at Stanford University. J Clin Oncol 14: 1282-1290, 1996.

30. Gustavsson A, Osterman B, Cavallin-Ståhl E: A systematic overview of radiation therapy effects in non-Hodgkin's lymphoma. Acta Oncol 42: 605-619, 2003. 